\title{
Alelos HLA más comunes y asociados con riesgo o protección en enfermedad renal crónica de etiología no determinada
}

\author{
Juan Carlos H. Hernández-Rivera, ${ }^{1}$ Mariana Salazar-Mendoza, ${ }^{2}$ María Juana Pérez-López, ${ }^{3}$ \\ Jaime González-Ramos, ${ }^{4}$ Ramón Espinoza-Pérez, ${ }^{5}$ Julio César Martínez-Álvarez, ${ }^{6}$ Miguel Trejo-Villeda ${ }^{1}$ \\ y Ramón Paniagua-Sierra ${ }^{1}$ \\ ${ }^{1}$ Instituto Mexicano del Seguro Social, Centro Médico Nacional Siglo XXI, Unidad de Investigación Médica en Enfermedades Nefrológicas; ${ }^{2}$ Instituto \\ de Seguridad y Servicios Sociales de los Trabajadores del Estado, Hospital Regional "Lic. Adolfo López Mateos", Servicio de Urgencias; ${ }^{3}$ Instituto \\ Mexicano del Seguro Social, Centro Médico Nacional La Raza, Servicio de Nefrología; ${ }^{4}$ Instituto Mexicano del Seguro Social, Centro Médico \\ Nacional La Raza, Laboratorio de Histocompatibilidad; ${ }^{2}$ nstituto Mexicano del Seguro Social, Centro Médico Nacional Siglo XXI, Unidad de \\ Trasplante Renal; ${ }^{6}$ Instituto Mexicano del Seguro Social, Centro Médico Nacional Siglo XXI, Laboratorio de Histocompatibilidad. Ciudad de México, \\ México
}

\section{Resumen}

Introducción: La enfermedad renal crónica representa parte del gasto en salud en general; una potencial etiología es la relacionada con variaciones, ausencia o presencia de algunos alelos del human leucocyte antigen (HLA). Método: Se realizó el análisis de 1965 reportes de HLA sin etiología determinada y de 1361 donadores renales. Se llevó a cabo tecnología Luminex con base en fluorimetría de flujo celular para los locus $A, B, D R B 1$ y DQA. Se realizó análisis con tablas de contingencia para determinar razón de momios (RM) e intervalos de confianza (IC). Se efectuó análisis cuantitativo. Resultados: De 101 alelos encontrados, 13 presentaron asociación, siete con riesgo para enfermedad renal crónica, de los cuales el más significativo fue HLA-DR17, con RM = 3.91 (IC $95 \%$ = 2.96-5.17), y el de mayor significación de protección fue HLA-DR9, con $R M=0.043$ (IC $95 \%=0.005-0.3224$ ). Conclusiones: Es necesario entender que las enfermedades renales pueden estar ligadas a procesos inmunológicos, en los que se tiene que conocer la asociación de la ausencia o presencia de algún alelo.

PALABRAS CLAVE: Enfermedad renal crónica. HLA. Receptores renales. Alelos.

\begin{abstract}
Introduction: Chronic kidney disease accounts for part of overall health expenditure; a potential etiology is related to variations, absence or presence of some human leukocyte antigen (HLA) alleles. Method: An analysis of HLA reports of 1965 kidney recipients with no determined etiology, and 1361 kidney donors was performed. It was carried out with Luminex based in cell flow fluorometry for the A, B, DRB1 and DQA loci. An analysis was performed with contingency tables in order to determine the odds ratio $(O R)$ and confidence intervals $(C l)$. Quantitative analysis was also carried out. Results: Of the 101 alleles found, 13 showed association, 7 with risk for chronic kidney disease, with the most significant being HLA-DR17 with an OR of 3.91 (95\% Cl = 2.96-5.17) and the one with the highest significance for protection being HLA-DR9, with an OR of 0.043 (95\% Cl =0.005-0.3224). Conclusions: It is necessary to understand that kidney diseases can be associated with yet unknown immune processes, where the association of the absence or presence of any allele should be known.
\end{abstract}

KEY WORDS: Chronic kidney disease. Human leukocyte antigen. Kidney recipients. Alleles.

Correspondencia:

Juan Carlos H Hernández-Rivera

E-mail: juancarloshhernandezrivera@ hotmail.com
Fecha de recepción: 28-01-2019

Fecha de aceptación: 21-02-2019

DOI: $10.24875 / G M M .19005033$
Gac Med Mex. 2019;155:243-248

Disponible en PubMed www.gacetamedicademexico.com 


\section{Introducción}

La incidencia y prevalencia de la enfermedad renal crónica (ERC) han aumentado significativamente debido al incremento de enfermedades cronicodegenerativas como la diabetes mellitus, la hipertensión arterial, la obesidad y la litiasis renal. ${ }^{1}$ En México, las glomerulopatías primarias y secundarias causan $12.7 \%$ de las ERC; ${ }^{2}$ una importante proporción de ellas tiene un componente autoinmune y en otras aún no se han dilucidado los aspectos básicos de su fisiopatología. En nuestro país es importante conocer la epidemiología de las ERC, cuya causa no se establece hasta en $50 \%$ de los pacientes no diabéticos que inician algún tratamiento de diálisis. ${ }^{3}$

Un número importante de enfermedades renales o sistémicas con afección renal (sin considerar a la diabetes) tiene componentes genético-inmunológico asociados con polimorfismo del complejo mayor de histocompatibilidad, específicamente con las moléculas HLA (human leucocyte antigen), involucradas en la regulación de procesos inmunológicos como el reconocimiento de antígenos, desarrollo de tolerancia inmunológica, comunicación celular, generación de citocinas o respuesta a ellas y factores asociados con inflamación y daño celular. ${ }^{4}$ Numerosas asociaciones HLA-enfermedad que conocemos actualmente no implican una liga directa con el alelo del gen, sino al desequilibrio del ligamento porque un alelo suele estar muy unido a otro perteneciente al locus que realmente tiene relación causal con la enfermedad. ${ }^{5}$

Existen ejemplos muy conocidos de enfermedades asociadas con el HLA, como la espondilitis anquilosante (con HLA-B27), ${ }^{6}$ la dermatitis herpetiforme (con HLA-A1) o la psoriasis asociada (con HLA-B13 y HLA-B17). ${ }^{7}$ Los estudios acerca de las relaciones de HLA con las enfermedades enfrentan tres problemas importantes:

- Los alelos asociados con una enfermedad también se encuentran en la población normal.

- Un solo alelo se puede asociar con más de una enfermedad.

- En $100 \%$ de los casos de una misma enfermedad no hay asociación con un solo alelo, debido a que subtipos de alelos o epítopes determinan la susceptibilidad a padecerla. ${ }^{8}$

Por otra parte, la asociación no siempre es positiva, por ejemplo, la lepra paracibacilar está relacionada con menor frecuencia de HLA-DQ04. ${ }^{9}$
Respecto a las enfermedades renales debidas a alteraciones genéticas, la glomerulopatía membranosa idiopática está ligada a M-type phospholipase A2 $R$ receptor, a depósitos glomerulares de $\lg \mathrm{G} 1$ e $\lg \mathrm{G} 3^{10}$ y a HLA-DR3 y DR23, con un riesgo relativo de 12.0.11 Otros alelos, como HLA-B18, se relacionan con riesgo aumentado de desarrollar glomerulopatía membranosa. ${ }^{12} \mathrm{La}$ glomerulonefritis posestreptoccócica se ha asociado con HLA-DRw4, ${ }^{13}$ HLA-DPA $1 * 02: 22$, HLA-DPB $1 * 05: 01^{14}$ y HLA-DRB1*03:11. ${ }^{15}$ En la glomerulonefritis antimembrana basal glomerular, el HLA relacionado es el DR2, en vínculo con DRB1*15:0116 y $\mathrm{DQB} * 06: 02 .^{17-20}$

En México no se cuenta con un registro de pacientes con ERC ni con un mecanismo de su referencia oportuna al nefrólogo, por lo cual se desconocen las causales estratificadas por factores demográficos como la edad, el sexo o las características raciales. A pesar de lo anterior, es posible inferir la importancia de los factores genéticos-inmunológicos en la epidemia de ERC mediante la tipificación de HLA en los pacientes que ingresan a un programa de trasplante renal. El HLA es un estudio básico en la selección del binomio receptor-donador, particularmente en donadores vivos, con el propósito de elegir al de mayor compatibilidad y menor riesgo de rechazo.

El objetivo de este estudio es analizar la potencial asociación entre ERC terminal y los alelos identificados en los estudios de HLA recabados durante la selección de los binomios donador-receptor en los programas de trasplante renal.

\section{Método}

Se analizó una cohorte retrospectiva de dos centros de trasplante entre el 1 de enero de 2010 y el 31 de diciembre de 2017.

Se analizaron los reportes de HLA de los pacientes en estudio como potenciales receptores renales y de sus potenciales donadores. Los criterios para los receptores fueron los establecidos por el comité local de cada centro de trasplante. Se excluyeron los pacientes con nefropatía diabética como causa de la ERC, así como los que no contaran con el HLA completo al momento de la captura de los datos.

Los reportes se obtuvieron de los expedientes médicos. La tipificación del HLA fue con tecnología Luminex con base en fluorimetría de flujo celular, empleando microesferas recubiertas con antígenos purificados HLA en los locus A, B, DRB1 y DQA; como método de tipificación se empleó el polymerase 
chain reaction sequence-specific oligonucleotide (PCR-SSO). Los datos se expresaron como frecuencias. Con la información de los cuatro locus se comparó la frecuencia de cada uno y se obtuvo el riesgo relativo por medio de tablas de contingencia de $2 \times$ 2 , con estimación de su razón de momios (RM) y su respectivo intervalo de confianza (IC), para valorar la significación estadística. Se realizó análisis cuantitativo por medio del programa SPSS versión 25 .

\section{Resultados}

Se recabaron los HLA de 1361 potenciales donadores renales (39\% era padre o madre); los 1965 potenciales receptores renales se agruparon por alelos en cada locus. Se procesaron los locus A, B, DRB1 y DQA para cada estudio de HLA. En la Tabla 1 se describen las frecuencias de los alelos, estratificados como "donadores" y "receptores".

Cabe resaltar que el alelo más común en el locus $\mathrm{A}$ fue el 02, presente en 1372 (34.91\%) receptores (se consideraron los dos haplotipos, por lo tanto, resultaron 3930 alelos en los receptores y 2722 alelos en los donadores) y 998 (36.66 \%) del alelo HLA-A2 en los donantes. En el locus B (el más polimórfico), el alelo más común fue el 35 , tanto para los receptores, con $830(21.11 \%)$, como para los donadores, con $618(22.7 \%)$. En el locus DRB1, el 04 fue el mayormente identificado, tanto en receptores como en donadores, con $1338(34.04 \%)$ y 864 (31.74\%), respectivamente. Por último, en el locus DQA, el menos polimórfico, el más común fue el 08, con 1395 (35.49 \%) en receptores y 918 (33.72\%) en donadores.

Respecto a los alelos de riesgo se encontraron siete asociados con riesgo y seis con protección para ERC. En total se identificaron 101 alelos, distribuidos por locus de la siguiente forma (Tabla 2):

- HLA-A fueron 28 alelos, 20 comunes (presentes en donadores y receptores), uno solo en donadores y siete en receptores; uno con significación estadística para riesgo, que fue el 11.

- HLA-B fueron 43 alelos, 30 comunes, siete solo en donadores y seis en receptores; los alelos 08 , 18 y 53 tuvieron significación de riesgo.

- HLA-DRB1 fueron 20 alelos, 16 comunes, tres en donadores y uno en receptor, con significancia para riesgo en tres casos $(04,11$ y 17), así como tres de protección $(08,09$ y 16).

- HLA-DQA fueron 10 alelos, nueve comunes y uno solo en donador; se identificaron dos alelos de protección para ERC (03 y 04).
Dos alelos llamaron particularmente la atención: uno de riesgo, el HLA-DR17, con RM = 3.91 (IC $95 \%=2.96-5.17$ ), y uno de protección con alto peso estadístico, el HLA-DR09, con RM $=0.043$ (IC $95 \%=0.005-0.3224)$.

\section{Discusión}

Pudimos corroborar que existen alelos de riesgo para presentar enfermedad renal, pero también de protección para disminuir las posibilidades de ERC; en total se recabaron 3326 HLA (1965 potenciales receptores renales y 1361 potenciales donadores). Los alelos más comunes por locus fueron similares a los encontrados en México por nuestro mismo grupo de trabajo: ${ }^{21}$ los alelos 2, 35, 4 y 3, respectivamente, fueron los más comunes para los locus A, B, DRB1 y DQA.

De los alelos de riesgo para ERC (siete), uno se encontró en el locus A (el 11), que se relacionó concretamente con citomegalovirus, Epstein-Barr y hepatitis autoinmune. ${ }^{22}$ En el locus $B$ se identificaron tres alelos de riesgo: el 8, relacionado con lupus eritematoso y nefropatía por $\lg A ;{ }^{23}$ el 18 , con glomerulopatía membranosa y lupus eritematoso sistémico:13 y el B17, aun cuando en la búsqueda en la literatura no encontramos una asociación con alguna enfermedad renal. Por último, en el locus DRB1 se encontraron tres alelos de riesgo: el alelo 4, relacionado con la glomerulonefritis posestreptocócica; ${ }^{13}$ el 11 , asociado con hepatitis $C$ (causa de glomerulopatía membranoproliferativa), glomerulonefritis, síndrome de Sjögren, lupus eritematoso y artritis reumatoidea; ${ }^{24}$ y el 17 , relacionado con lupus e hipertrofia cardiovascular. ${ }^{25,26}$

Respecto a los seis alelos de protección significativos, no encontramos estudios que pudieran establecer un paralelismo con los resultados de nuestro trabajo (Tabla 2).

En numerosos pacientes renales en protocolo de trasplante no se conocía la etiología (más de $95 \%$ ), por lo que fue difícil asumir la relación, sin embargo, se pudo establecer la significación estadística de riesgo y protección en cada caso, específicamente en 13 de los 101 alelos, siete de riesgo y seis de protección, información con la que se podría determinar, aunque no con certeza, las potenciales causas en los pacientes con estos alelos y así realizar diagnósticos más tempranos de enfermedades renales y comprobar dichas asociaciones con mayor precisión. 
Tabla 1. Diferencia de alelos en 1965 potenciales receptores renales y 1361 donadores

\begin{tabular}{|c|c|c|c|c|c|c|c|c|c|c|c|c|c|c|c|}
\hline \multicolumn{4}{|c|}{ Locus A } & \multicolumn{4}{|c|}{ Locus B } & \multicolumn{4}{|c|}{ Locus DRB1 } & \multicolumn{4}{|c|}{ Locus DQA } \\
\hline \multicolumn{2}{|c|}{ Donadores } & \multicolumn{2}{|c|}{ Receptores } & \multicolumn{2}{|c|}{ Donadores } & \multicolumn{2}{|c|}{ Receptores } & \multicolumn{2}{|c|}{ Donadores } & \multicolumn{2}{|c|}{ Receptores } & \multicolumn{2}{|c|}{ Donadores } & \multicolumn{2}{|c|}{ Receptores } \\
\hline 01 & 110 & 01 & 170 & 06 & 1 & 07 & 130 & 01 & 149 & 01 & 207 & 01 & 4 & 01 & 9 \\
\hline 02 & 998 & 02 & 1372 & 07 & 99 & 08 & 105 & 03 & 36 & 03 & 47 & 02 & 258 & 02 & 393 \\
\hline 03 & 76 & 03 & 133 & 08 & 51 & 13 & 36 & 04 & 864 & 04 & 1338 & 03 & 361 & 03 & 458 \\
\hline 11 & 73 & 07 & 1 & 13 & 23 & 14 & 50 & 06 & 1 & 06 & 2 & 04 & 297 & 04 & 348 \\
\hline 19 & 1 & 08 & 1 & 14 & 44 & 15 & 106 & 07 & 166 & 07 & 214 & 05 & 241 & 05 & 351 \\
\hline 23 & 48 & 09 & 1 & 15 & 72 & 16 & 1 & 08 & 585 & 08 & 721 & 06 & 231 & 06 & 333 \\
\hline 24 & 449 & 11 & 114 & 18 & 51 & 18 & 119 & 09 & 16 & 10 & 36 & 07 & 392 & 07 & 614 \\
\hline 25 & 14 & 13 & 3 & 27 & 48 & 27 & 52 & 10 & 25 & 11 & 176 & 08 & 918 & 08 & 1395 \\
\hline 26 & 55 & 23 & 60 & 30 & 1 & 32 & 1 & 11 & 90 & 12 & 18 & 09 & 19 & 09 & 29 \\
\hline 28 & 2 & 24 & 684 & 31 & 1 & 35 & 830 & 12 & 9 & 13 & 209 & 15 & 1 & & \\
\hline 29 & 80 & 25 & 25 & 33 & 1 & 37 & 19 & 13 & 140 & 14 & 411 & & & & \\
\hline 30 & 95 & 26 & 68 & 35 & 618 & 38 & 58 & 14 & 267 & 15 & 180 & & & & \\
\hline 31 & 167 & 28 & 1 & 37 & 10 & 39 & 682 & 15 & 136 & 16 & 23 & & & & \\
\hline 32 & 40 & 29 & 105 & 38 & 41 & 40 & 102 & 16 & 152 & 17 & 324 & & & & \\
\hline 33 & 57 & 30 & 131 & 39 & 474 & 41 & 31 & 17 & 61 & 18 & 13 & & & & \\
\hline 34 & 5 & 31 & 261 & 40 & 79 & 42 & 11 & 18 & 14 & 19 & 1 & & & & \\
\hline 36 & 2 & 32 & 52 & 41 & 22 & 44 & 201 & 51 & 1 & 103 & 10 & & & & \\
\hline 66 & 10 & 33 & 82 & 42 & 11 & 45 & 52 & 53 & 1 & & & & & & \\
\hline 68 & 430 & 34 & 10 & 44 & 135 & 46 & 4 & 103 & 9 & & & & & & \\
\hline 69 & 5 & 35 & 1 & 45 & 24 & 47 & 1 & & & & & & & & \\
\hline \multirow[t]{15}{*}{74} & 5 & 36 & 7 & 47 & 1 & 48 & 136 & & & & & & & & \\
\hline & & 39 & 2 & 48 & 103 & 49 & 49 & & & & & & & & \\
\hline & & 66 & 16 & 49 & 37 & 50 & 31 & & & & & & & & \\
\hline & & 68 & 618 & 50 & 17 & 51 & 221 & & & & & & & & \\
\hline & & 69 & 2 & 51 & 153 & 52 & 103 & & & & & & & & \\
\hline & & 74 & 7 & 52 & 96 & 53 & 48 & & & & & & & & \\
\hline & & 80 & 3 & 53 & 18 & 55 & 15 & & & & & & & & \\
\hline & & & & 54 & 1 & 56 & 9 & & & & & & & & \\
\hline & & & & 55 & 11 & 57 & 32 & & & & & & & & \\
\hline & & & & 56 & 7 & 58 & 27 & & & & & & & & \\
\hline & & & & 57 & 17 & 60 & 50 & & & & & & & & \\
\hline & & & & 58 & 18 & 61 & 238 & & & & & & & & \\
\hline & & & & 59 & 1 & 62 & 192 & & & & & & & & \\
\hline & & & & 60 & 24 & 63 & 18 & & & & & & & & \\
\hline & & & & 61 & 143 & 64 & 28 & & & & & & & & \\
\hline
\end{tabular}


Tabla 1. Diferencia de alelos en 1965 potenciales receptores renales y 1361 donadores (Continuación)

\begin{tabular}{|c|c|c|c|c|c|c|c|c|c|}
\hline \multicolumn{2}{|c|}{ Locus A } & \multicolumn{4}{|c|}{ Locus B } & \multicolumn{2}{|c|}{ Locus DRB1 } & \multicolumn{2}{|c|}{ Locus DQA } \\
\hline \multirow[t]{2}{*}{ Donadores } & Receptores & \multicolumn{2}{|c|}{ Donadores } & \multicolumn{2}{|c|}{ Receptores } & Donadores & Receptores & Donadores & Receptores \\
\hline & & 62 & 151 & 65 & 71 & & & & \\
\hline & & 63 & 10 & 68 & 2 & & & & \\
\hline & & 64 & 11 & 69 & 1 & & & & \\
\hline & & 65 & 51 & 71 & 10 & & & & \\
\hline & & 70 & 2 & 72 & 19 & & & & \\
\hline & & 71 & 10 & 73 & 7 & & & & \\
\hline & & 72 & 11 & 75 & 7 & & & & \\
\hline & & 73 & 5 & 78 & 10 & & & & \\
\hline & & 75 & 11 & 81 & 3 & & & & \\
\hline & & 78 & 4 & 82 & 2 & & & & \\
\hline & & 81 & 3 & & & & & & \\
\hline
\end{tabular}

Tabla 2. Alelos de riesgo y protección en enfermedad renal crónica

\section{Locus}

\section{Alelo Razón de} momios

\section{Intervalo de} confianza

De riesgo para ERC

\begin{tabular}{|l|l|l|l|}
\hline HLA-A & 11 & 1.61 & $1.14-2.27$ \\
\hline HLA-B & 08 & 1.42 & $1.02-2.01$ \\
\hline & 18 & 1.63 & $1.17-2.27$ \\
\hline HLA-DRB1 & 04 & 1.11 & $1.07-3.20$ \\
\hline & 11 & 1.37 & $1.002-1.23$ \\
\hline & 17 & 3.91 & $1.06-1.78$ \\
\hline
\end{tabular}

De protección para ERC

\begin{tabular}{|l|c|c|c|}
\hline HLA-B & 52 & 0.73 & $0.55-0.97$ \\
\hline HLA-DRB1 & 08 & 0.82 & $0.72-0.92$ \\
\hline & 09 & 0.043 & $0.005-0.3224$ \\
\hline & 16 & 0.09 & $0.06-0.15$ \\
\hline HLA-DQA & 03 & 0.86 & $0.74-0.99$ \\
\hline & 04 & 0.79 & $0.67-0.93$ \\
\hline
\end{tabular}

ERC=enfermedad renal crónica

La principal limitante del estudio estribó en que no fue posible identificar la asociación entre los alelos de HLA y la causa de la ERC, pues lamentablemente en más de $95 \%$ de los pacientes se desconocía la causa al no tener las biopsias renales por la tardanza con que acudieron los pacientes a atención médica. Se excluyeron los pacientes con nefropatía diabética.

\section{Conclusiones}

Es imprescindible conocer las causas que originan la ERC como una línea de acción para disminuir la morbilidad y mortalidad por esta entidad. El origen inmunológico no debe ser minimizado ni la presencia, ausencia y asociación de los alelos, tanto por el riesgo como por la protección que pueden implicar. En total se encontraron 13 alelos relacionados con riesgo-protección: siete de riesgo $(6.9 \%)$, con significación estadística, y seis de protección (5.9\%) para la presencia de ERC. Dos de los 13 alelos tuvieron particular significación estadística: HLA-DR17, para riesgo, y HLA-DR, para protección.

En cuanto a la glomerulopatía, conocer la causa de la ERC permitiría lo siguiente:

- Mejorar la certeza de la asociación HLA-glomerulopatía, con base en el resultado histopatológico de una biopsia renal y la determinación del HLA, como parte del estudio de un potencial receptor renal.

- Estimar los riesgos de recidiva de la glomerulopatía postrasplante.

\section{Bibliografía}

1. Dehesa E. Enfermedad renal crónica; definición y clasificación. El Residente. 2008;3:73-78.

2. Méndez-Durán A, Méndez-Bueno F, Tapia-Yáñez T, Muñoz-Montes A, Aguilar-Sánchez L. Epidemiología de la insuficiencia renal crónica en México. Diálisis y Trasplante. 2001;31:7-11.

3. Flores J. Enfermedad renal crónica: epidemiologia y factores de riesgo. Rev Med Clin Condes. 2010;21:502-507.

4. Jadue N, González A. Inmunopatogenia de las enfermedades autoinmunes. Rev Med Clin Las Condes. 2012;23:464-472. 
5. Morera-Barrios LM, Verdura-González TE, Martínez-Legón Z Ricardo-Lorenzo R. Asociación de antígenos HLA y enfermedades oftalmológicas. Rev Cubana Hematol Inmunol Hemoter. 2001;17:7-18.

6. Tolosa A. Primer catálogo de enfermedades asociadas con variaciones en los genes de los antígenos leucocitarios humanos. España: Genética Médica; 2017.

7. Corrales P, Corrales A, Carias S. Asociación entre HLA y enfermedad estado actual y perspectivas. Rev Med Hondur. 1985;53:104-118.

8. Fernández J. HLA y enfermedad. Rev Med Hondur. 1991;51:151-154.

9. Fabiana P, Cech N, Fontan C, Giménez M, Lodeiro N, Marinic K Molinari M, Sotelo M, Habegger A. Papel de las moléculas HLA-DR y HLA DQ en la lepra en la provincia del Chaco, Argentina. Enferm Infecc Microbiol Clin. 2007;25:627-631.

10. Nachman P, Jannette J Falk R. Primary glomerular disease. En: Skorecki K, Chertow GM, Marsden PA, Taal MW. Brenner \& Rector's. The Kidney. EE. UU.: Elsevier; 2012

11. Klouda PT, Manos J, Acheson EJ, Dyer PA, Goldby FS, Harris R, et al Strong association between idiopathic membranous nephropathy and HLA-DRW3. Lancet. 1979:2:770-771.

12. Dyer PA, Klouda PT, Harris R, Mallick NP. Properdin factor B alleles in patients with idiopathic membranous nephropathy. Tissue Antigens. 1980;15:505-507.

13. Layrisse Z, Rodríguez-Iturbe B, García-Ramírez R, Rodríguez A Tiwari J. Family studies of the HLA system in acute post-streptococcal glomerulonephritis. Hum Immunol. 1983;7:177-185.

14. Mori K, Sasazuki T, Kimura A, Ito Y. HLA-DP antigens and poststreptococcal acute glomerulonephritis. Acta Pediatr. 1996;85:916-918.

15. Bakr A, Mahmoud LA, Al-Chenawi F, Salah A. HLA-DRB1* alleles in Egyptian children with post-streptococcal acute glomerulonephritis. Pediatr Nephrol. 2007;22:376-379.

16. Fisher M, Pusey CD, Vaughan RW, Rees AJ. Susceptibility to anti-glomerular basement membrane disease is strongly associated with HLADRB1 genes. Kidney Int. 1997;51:222-229.
17. Huey B, McCormick K, Capper J, Ratliff C, Colombe BW, Garovoy MR, et al. Associations of HLA-DR and HLA-DQ types with anti-GBM nephritis by sequence-specific oligonucleotide probe hybridization. Kidney Int. 1993;44:307-312.

18. Dunckley $\mathrm{H}$, Chapman JR, Burke J, et al. HLA-DR and -DQ genotyping in anti-GBM disease. Dis Markers. 1991;9:249-256.

19. Burns AP, Fisher M, Li P, Pusey CD, Rees AJ. Molecular analysis of HLA class II genes in Goodpasture's disease. QJM. 1995;88:93-100.

20. Kitagawa W, Imai H, Komatsuda A, Maki N, Wakui H, Hiki $Y$, et al. The HLA-DRB1*1501 allele is prevalent among Japanese patients with anti-glomerular basement membrane antibody-mediated disease. Nephrol Dial Transplant. 2008:23:3126-3129.

21. Hernández-Rivera JCH, González-Ramos J, Pérez-López MJ, Carmona-Becerril A, Escárcega-Vázquez A, Cruz-Santiago J, et al. The most common HLA antigens found in patients with renal transplants at the specialties hospital of "La Raza" medical center, México. Transplant Proc. 2016;48:572-574.

22. Contreras G, Lenz O, Pardo V, Borja E, Cely C, Iqbal K, et al. Outcome in African Americans and Hispanics with lupus nephritis. Kidney Int. 2006;69:1846-1851.

23. Appel GB, Jayne D. Lupus nephritis. En: Johnson R, Floege J, Feehaly J, editores. Comprehensive clinical nephrology. EE. UU: Elsevier; 2010.

24. Gendi NS, Welsh KI, Van-Venrooij WJ, Vancheeswaran R, Gilroy J, Black CM. HLA type as a predictor of MCTD differentiation. Ten year clinical and immunogenetic follow-up of 46 patients. Arthritis Rheum. 1995;38:259-266.

25. Diamantopoulos EJ, Andreadis EA, Vassilopoulos CV, Vlachonikolis IG, Tarassi KE, Chatzis N, et al. Association of specific HLA phenotypes with left ventricular mass and carotid intima-media thickness in hypertensives. Am J Hypertens. 2001;14:632-636.

26. Waldman M, Madaio MP. Pathogenic autoantibodies in lupus nephritis. Lupus. 2005;14:19-24. 